Recepción: 20 / 10 / 2017

Aceptación: 9/ 11 / 2017

Publicación: 15 / 12 / 2017

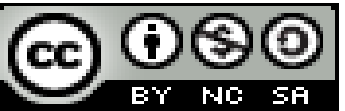

Ciencias Sociales y políticas

Artículo Científico

\title{
Marketing Online: Un enfoque global
}

Online Marketing: A global approach

\section{Marketing on-line: uma abordagem global}

Fernando E. Viteri-Luque ${ }^{\mathrm{I}}$

fernando.viteril@ug.edu.ec

Luis A. Herrera-Lozano II

luis.herreral@ug.edu.ec

Antonio F. Bazurto-Quiroz III

antonio.bazurtoq@ug.edu.ec

Correspondencia: fernando.viteril@ug.edu.ec

\footnotetext{
I Docente de la Universidad de Guayaquil, Guayaquil, Ecuador.

II Docente de la Universidad de Guayaquil, Guayaquil, Ecuador. 


\section{Resumen}

El marketing digital o marketing online es el desarrollo de estrategias de publicidad y comercialización a través de los medios digitales, en las cuales todas las técnicas que se emplean en el mundo off-line (web) son imitadas y traducidas al mundo online, es por ello que, en el marketing digital aparecen, por ejemplo, nuevas herramientas, como los buscadores como google, las nuevas redes sociales y la posibilidad de mediciones digitales de rentabilidad de cada una de las estrategias empleadas (IIEMD, n.ed) .Las innovaciones en el área del marketing digital están fundamentadas básicamente en la web 2.0, debido a que esta nace con la posibilidad de compartir información fácilmente por medio de las redes sociales y a las nuevas tecnologías de información, que permiten a su vez el intercambio casi instantáneo de información que antes eran imposible compartir con tanto alcance y velocidad como en el mundo online (Tirado, 2013). El marketing digital emplea el internet no sólo como medio para encontrar información sino como comunidad, donde hay relaciones constantemente y feedback con los usuarios de diferentes partes del mundo, por lo que busca resaltar la opinión de los usuarios de manera online (IIEMD, n.ed).

Palabras Claves: Marketing, Marketing digital, satisfacción del cliente, consumidor, tendencias. 


\begin{abstract}
Digital marketing or online marketing is the development of advertising and marketing strategies through digital media, in which all the techniques used in the off-line world (web) are imitated and translated into the online world. In digital marketing, for example, new tools appear, such as search engines such as google, new social networks and the possibility of digital profitability measurements of each of the strategies employed (IIEMD, n.ed). Innovations in the area of digital marketing are basically based on web 2.0, because this is born with the possibility of sharing information easily through social networks and new information technologies, which in turn allow the almost instantaneous exchange of information that previously were impossible to share with as much scope and speed as in the online world (Tirado, 2013). Digital marketing uses the internet not only as a means to find information but as a community, where there are constant relationships and feedback with users from different parts of the world, so it seeks to highlight the opinion of users online (IIEMD, n. ed).
\end{abstract}

Keywords: Marketing, digital marketing, customer satisfaction, consumer, trends. 


\section{Introducción.}

Las empresas y la forma como establecen relaciones comerciales han ido evolucionando en las últimas décadas y con ellas el hombre ha desarrollado nuevas maneras de crear relaciones económicas, en pro de comercializar el producto de su trabajo. En un principio, el empresario no reparaba en los intereses y expectativas que el consumidor pudiese tener. Por lo tanto, las relaciones económicas entre vendedores y consumidores se sostenían sobre criterios unidireccionales, toda vez que el mercado, inicialmente, tenía un nivel de alcance circunscrito a las ciudades y el hombre no contaba con variadas alternativas de selección. Este debía conformarse (Chiavenato, 2001). Junto con la revolución industrial evolucionaron los mercados. Estos incrementaron su tamaño y el hombre igualmente desarrolló nuevas maneras de establecer relaciones comerciales que transcendían las fronteras entre países (Gutierrez, 2010).

La modernidad, replanteó todas las maneras de pensar en cuanto a los procesos de interacción comercial se refiere, abriéndole paso progresivamente a enfoques centrados en los gustos y preferencias del consumidor (Tirado, 2013). El marketing llegó inicialmente, de la mano de las grandes empresas, para posteriormente ser asumido por el resto de los pequeños empresarios como una necesidad en medio de la incertidumbre y el cambio (Ph.D McCarthy E., 2001).

La globalización, como fenómeno mundial ha sido capaz de homogeneizar la cultura sobre la base de la revolución de la información y la tecnología (MktS.A., 2015). La sociedad se comunica en tiempo real, las barreras físicas y geográficas son cosa del pasado (Inboundcycle, 2014). El intercambio, es increíblemente rápido. Los mercados, ahora se comportan de forma dinámica y están erigidos sobre principio de la complejidad, lo que hace mucho más difícil su estudio y comprensión (Tirado, 2013). Es por ello que este artículo aspira aclarar algunos términos y aplicaciones de 
Fernando E. Viteri-Luque; Luis A. Herrera-Lozano; Antonio F. Bazurto-Quiroz

herramientas vinculadas al marketing digital como un enfoque global dentro de la estrategia de comercialización que debe contemplar cualquier empresa en la actualidad.

\section{Materiales y métodos}

\section{Marketing}

Es la disciplina encargada de analizar el comportamiento de los consumidores y del entorno (mercado) en donde se desenvuelven los clientes, con el objetivo de captar, retener y fidelizar a los clientes a través de la satisfacción de sus necesidades, lo que impacta de manera directa el proceso de comercialización en una organización, debido a que el marketing lo que hace es considerar una necesidad de la clientela y, a partir de ella, diseña, pone en marcha y verifica cómo funciona la comercialización de los productos o servicios de la empresa (Ph.D McCarthy E., 2001) Es una variedad de estrategias y herramientas lo que permiten al marketing posicionar una marca o un producto en la mente del comprador.

De acuerdo a los expertos, tal y como lo señala (Tirado, 2013), el marketing (mercadeo o mercadotécnica) es:

Según Philip Kotler consiste en un proceso administrativo y social gracias al cual determinados grupos o individuos obtienen lo que necesitan o desean a través del intercambio de productos o servicios.

Jerome McCarthy opina que es la realización de las actividades que pueden ayudar a que una empresa consiga las metas que se ha propuesto, pudiendo anticiparse a los deseos de los consumidores y desarrollar productos o servicios aptos para el mercado. 
Por su parte John A. Howard, quien trabaja en la Universidad de Columbia, asegura que está convencido de que el marketing consiste en un proceso en el que es necesario comprender las necesidades de los consumidores, y encontrar qué puede producir la empresa para satisfacerlas. Y Al Ries y Jack Trout, opinan que marketing es sinónimo de "guerra" donde cada competidor debe analizar a cada "participante" del mercado, comprendiendo sus fuerzas y debilidades y trazar un plan a fin de explotarlas y defenderse.

Según la American Marketing Asociation (A.M.A.), el marketing es una forma de organizar un conjunto de acciones y procesos a la hora de crear un producto "para crear, comunicar y entregar valor a los clientes, y para manejar las relaciones" y su finalidad es beneficiar a la organización satisfaciendo a los clientes.

\section{Marketing Digital}

El marketing ha ido evolucionando junto a la tecnología, lo que implica que los espacios de interacción trasciendan el ámbito físico tradicional, entendiendo por este último, el lugar en el que se desarrollan las operaciones de compra y venta para dar paso a acciones de interacción en un plano simbólico, en el que el usuario o el cliente no precisan de lo presencial para comprar una idea, un servicio o un bien determinado (Ph.D McCarthy E., 2001). A través del uso del internet, las empresas tienen a su disposición una gama amplia de herramientas que le permiten establecer procesos comunicacionales con sus clientes en pro de crear fidelización, captar o retener clientes. Como toda herramienta de gran poder e impacto a nivel mundial, el internet requiere conocer los 
pros y contra a fin de lograr aprovechar en toda su dimensión las bondades del marketing online. En este sentido, una de las principales bondades que ofrece el marketing digital implica una inversión pequeña, pero si se debe contar con alguien calificado que goce de gran inteligencia en su manejo. En virtud de lo anterior, es vital señalar que las estrategias de marketing digital deben ser vista por la empresa como un complemento de las estrategias de marketing general establecidas por la organización y el equipo de mercadeo encargado de tales fines (Chiavenato, 2001).

Figura.1. Marketing Digital

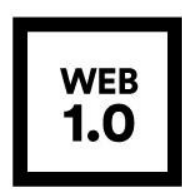

La primera se basa en la web 1.0, que no difiere de la utilización de medios tradicionales. Su mayor característica es la imposibilidad de comunicación y exposición de los usuarios. Solamente la empresa tiene el control de aquello que se publica sobre sí misma.

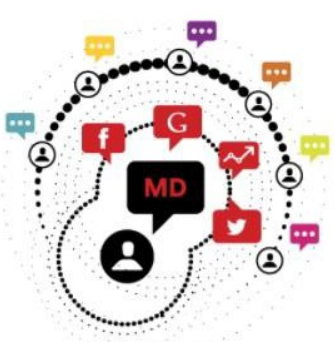

WEB 2.0

Fuente: (MktS.A., 2015)

En virtud de lo que implica el marketing web 2.0 como se pudo observar en la figura No. 1, cabe destacar que lo más utilizado en la actualidad es: Páginas web, comercio por correo electrónico, empleo de las redes sociales, optimización para buscadores y boletines digitales. Este ultimo enfocado a realizar mercado interno enfocado a los trabajadores, como pieza fundamental en el éxito de cualquier objetivo que se trace la organización en términos de calidad (Ph.D McCarthy E., 2001). Si bien es cierto que el objetivo fundamental del marketing sustentado en la plataforma de internet radica en incrementar progresivamente el número de usuarios $\mathrm{y}$, por ende, los posibles clientes de la empresa, ya que la meta que se persigue es la fidelización de los clientes para que estos conviertan 
de clientes potenciales y/o esporádicos a permanentes (MktS.A., 2015). El marketing realmente lo que busca es el establecimiento de una relación directa entre usuario y empresa comprendiendo en todo momento que este punto, demanda de inversiones moderadas por parte de las organizaciones (Zuritavas, 2017).

En cuanto a la captación de clientes vía online se refiere (Ph.D McCarthy E., 2001) indica que algunas de las herramientas más importantes para la captación usuarios en internet son:

- Lenguaje SEO: esta palabra significa Search Engine Optimization. Es decir, se trata de criterios de optimización para que una web sea ubicada con facilidad por los motores de búsqueda y se posicione entre los primeros puestos del buscador.

- SEM: esta palabra significa Search Engine Marketing. Es decir, se trata de marketing de buscadores, entendiendo por este la publicidad paga y que evidenciamos cada día que navegamos mediante una serie de anuncios o patrocinios que observamos sin nosotros quererlo (p.43).

3. Beneficios de algunas herramientas empleadas en el marketing digital

Según (Zuritavas, 2017), las principales bondades son:

\section{Beneficios de Facebook}

- Dar a conocer y fortalecer una marca. 
Fernando E. Viteri-Luque; Luis A. Herrera-Lozano; Antonio F. Bazurto-Quiroz

- Alcanzar nuevos mercados a través de contenidos de valor para tu audiencia que permitirá aumentar tu número de seguidores.

- Es una plataforma que te permite informar al instante de nuevos productos y servicios al segmento de tu interés ya que tienes el poder de segmentar la audiencia al que llegarán tus anuncios.

- Es un aliado para generar leads, incrementar tráfico a tu website y brindar un canal de comunicación directa con tus potenciales clientes.

\section{Beneficios de Instagram}

- Ideal para dar a conocer al mundo tu marca o fortalecerla.

- Comunicarte con personas afines a tus productos o servicios de una manera más cercana y muy visual.

- No necesitas ser un fotógrafo profesional para publicar tus imágenes en esta red social, Instagram cuenta con una serie de filtros que serán un aliado para que tus publicaciones tengan un efecto WOW.

- Los usos del hashtag en tus publicaciones harán más fácil que las personas conecten con tu marca si compartes sus mismos gustos o estilos de vida.

\section{Beneficios de LinkedIn}

- Es un escaparate donde podrás exhibir tus conocimientos y habilidades profesionales. 
- Mediante el perfil de la empresa podrás promocionar tus productos y servicios.

- Identificar perfiles de posibles clientes y verificar quien de tu red de contactos te los podría presentar.

- Compartir contenido de valor de carácter profesional con tu red de contactos y posicionarte como un especialista en tu sector.

\section{Beneficios de Twitter}

- Plataforma para difundir noticias de una manera rápida.

- Mediante los hashtags un tema se puede hacer un trendy topic en tu área geográfica que podría ser relevante para tu audiencia.

- Excelente opción para encontrar referentes locales o internacionales en tu industria, que comparten información de valor para su comunidad.

\section{Resultados.}

\section{Nuevas tendencias}

En un entorno cambiante y tan competitivo como el ecuatoriano, el consumidor ha desarrollado en los últimos tiempos la prudencia y consciencia a la hora de elegir un producto o servicio independientemente de la modalidad de compra; entiéndase, si el proceso de comercialización es presencial o virtual. La relación con el cliente es en consecuencia la piedra angular del marketing digital y se basa en excelencia del producto junto a la tecnología que acompaña en proceso logística que implica establecer el comercio electrónico o e-commerce (Tirado, 2013). 
Pese a que no existe una estadística exacta en relación al tema en Ecuador, se puede inferir que existe un incremento sostenido y significativo de la cantidad de personas interactúan a través de internet y que a su vez compran y venden productos por este medio, ya que todas las plataformas que posibilitan el comercio por esta vía cuentan con mecanismos valorativos sobre la confiabilidad del vendedor, que brindan seguridad al comprador y finalmente, la población ecuatoriana ha acumulado suficiente experiencia para comprender cómo funcionan estas plataformas. En este sentido, se hace propicia la ocasión para señalar algunas de las nuevas tendencias que existen en materia de mercadeo digital, las cuales se mencionan a continuación:

a) Marketing de contenido: Los usuarios son cada vez más exigentes y demandan noticias más elaboradas y escritas por expertos y profesionales que hayan recurrido a fuentes fiables, es por ello que ahora se trabaja en formato largo, fuentes fiables y mejor calidad de la información que se publica (Pélaez, 2017)

b) Influenciadores: Gracias a los prescriptores, las marcas trasladan sus valores y su lado más humano a seguidores que empatizan con esas personas, la falta de transparencia suscita suspicacia entre los compradores. Su capacidad de prescripción se ha fundamentado en la credibilidad que les conceden sus seguidores, quienes son cada vez más conscientes de que, por lo habitual, existe una contraprestación económica cuando hablan de ciertos productos o servicios. Es por ello que se busca más transparencia en este aspecto (Pélaez, 2017).

c) Vivimos en el móvil: Al consumidor literalmente le ha dejado de interesar lo que sucede en su entorno inmediato, ya que ese entorno se ha trasladado a un espacio menos tangible: el móvil. Según (Pélaez, 2017) señala que una persona ve el móvil más de 80 veces al día; es 
por ello que (Zuritavas, 2017) estima que en el 2020, el 70\% de la población utilizará el smartphone, según publicó Ericsson. Son estos datos los que propician una señal clara de hacia donde se deben abocar las estrategias de marketing, si es que estas aspiran ser exitosas. En virtud de esto, es que se debe apuntar hacia experiencias digitales mucho más avanzadas gracias a la realidad virtual, es decir render, videos de 360 grados, crear un viaje al consumidor personalizado y satisfactorio en todas sus fases (Pélaez, 2017).

d) Cambios en las redes sociales: No es que Facebook pierda impacto ante sus seguidores, sino que Instagram y Snapchat encajan mejor con las nuevas generaciones de usuario, ya que satisfacen la necesidad apremiante de los más jóvenes por aprehender el mundo a través de imágenes (Pélaez, 2017).

e) Monitoriza y segmenta tus audiencias: Pese a que parece bastante obvio que, antes de crear cualquier campaña o publicación, se debe conocer a la audiencia objetivo y saber cuáles son sus necesidades o cómo perciben la marca de la organización. Se hace vital mencionarlo ya que, si no se miden las reacciones de una campaña, resulta sumamente complicado llevar a cabo una estrategia de éxito. Cuanto más se conozca al cliente (audiencia), más se podrá hacer por ella (Pélaez, 2017).

\subsection{Marketing de afiliación}

El marketing de afiliación aprovecha las plataformas que no le son propias para que la empresa pueda proyectar su propia marca. En este sentido, dirige mensajes o bien, a una red de afiliados o a un conjunto de empresas a las cuales se les paga por colocar sus anuncios (Ph.D McCarthy E., 2001). 


\subsection{E-mailing}

Esta modalidad consiste en el envío de correos electrónicos montados sobre una plataforma publicitaria, que le imprime orden a la formación. Esta estrategia de marketing es de carácter masivo (Ph.D McCarthy E., 2001). Por lo tanto, sus resultados dependen de la cantidad de envíos vs la cantidad de leídos (Gutierrez, 2010).

\section{Conclusiones.}

El marketing digital es una estrategia imprescindible para las marcas por la gran oportunidad de crecimiento, posicionamiento y difusión que representan. Los clientes potenciales, o al menos su gran mayoría, están constantemente conectados a internet desde el ordenador y, en los últimos años, también a través de sus teléfonos móviles o tablets (MktS.A., 2015). Este contexto ha propiciado un amplio abanico de ventajas del marketing digital, debido a que presenta según (Inboundcycle, 2014):

- Costes asequibles. El marketing online es accesible en términos de presupuesto, sobre todo si se los compara con los canales de marketing tradicionales como, por ejemplo, la televisión, la radio o la prensa.

- Mayor capacidad de control, optimización y corrección de las campañas debido a la recogida y posibilidad de consulta en tiempo real de los resultados obtenidos, además, de manera exacta.

- Gran flexibilidad y dinamismo. Con la posibilidad de realizar testeos y cambios sobre la marcha en función de los resultados obtenidos y el comportamiento de los usuarios respecto a una campaña. 
- Permite una segmentación muy específica, personalizada y precisa.

En una campaña de marketing online, la empresa puede segmentar sus campañas teniendo en cuenta los datos sociodemográficos, psicológicos de los usuarios, así como según su comportamiento en internet.

- Permite una medición exacta de la campaña. Resultados obtenidos, beneficios, retorno de la inversión (ROI), etc.

\section{Referencias.}

Chiavenato, I. (2001). Administración: Teoría, proceso y práctica. México: Mc Graw-Hill.

Gutierrez, H. (2010). Calidad total y productividad. México: McGraw Hill Interamaricana, 3era edición.

IIEMD, I. I. (n.ed). Marketing digital. recuperado de: https://iiemd.com/marketing-digital/que-esmarketing-digital.

Inboundcycle. (2014). Que es el marketing digital o marketing online. recuperado de: https://www.inboundcycle.com/blog-de-inbound-marketing/que-es-el-marketing-digital-omarketing-online.

MktS.A., M. (2015). Marketing digital. recuperado de: https://www.mdmarketingdigital.com/quees-el-marketing-digital.php.

Pélaez, I. (2017). 5 tendencias del marketing en 2018 recuperado de: https://www.brandwatch.com/es/blog/tendencias-del-marketing/.

Ph.D McCarthy E., P. P. (2001). Marketing: un enfoque global. México: Mc Graw- Hill , 13a edición.

Tirado, D. M. (2013). Fundamentos de Marketing. Castellón de la Plana: Publicaciones de la Universidad de Jaumé.

Zuritavas, D. (2017). Tips sobre el marketing digital. recuperado de: https://dianazuritavas.wordpress.com/2017/09/21/ecuador-cuales-son-las-redes-socialesmas-utilizadas/. 